\title{
A diffusion tensor magnetic resonance imaging study of brain tissue from patients with migraine
}

\author{
M A Rocca, B Colombo, M Inglese, M Codella, G Comi, M Filippi
}

J Neurol Neurosurg Psychiatry 2003;74:501-503

Objective: To measure in vivo, using diffusion tensor magnetic resonance imaging (DT-MRI) the extent of pathological damage of normal appearing brain tissue (NABT) from patients with migraine.

Methods: Dual echo and DT-MRI scans of the brain were acquired from 34 patients with migraine and 17 sex and age matched healthy volunteers. Mean diffusivity (MD) and fractional anisotropy (FA) histograms of the NABT were obtained from all subjects and the histograms' peak heights and average NABT MD and FA measured. When present, average $M D$ and $F A$ values of $T 2$ visible lesions were also measured.

Results: In comparison with healthy volunteers, patients with migraine had lower MD histogram peak height $(p=0.02)$ of the NABT. No differences were found in FA histogram derived metrics between migraine patients and healthy subjects. No difference was found for any MD and FA histogram derived metrics between migraine patients with and without brain MRI lesions, and between patients with and without aura.

Conclusions: This study shows that, although brain damage may extend beyond T2 weighted abnormalities in patients with migraine, the severity of these "occult" changes is mild compared with that found in other diseases associated with white matter abnormality.

$\mathrm{U}$ sing T2 weighted magnetic resonance imaging (MRI), brain white matter abnormalities have been detected from patients with migraine with a frequency ranging from $12 \%$ to $46 \% .^{1-5}$ Although the exact nature of these abnormalities is still unclear, they are likely to be the consequence of ischaemic damage secondary to the repeated regional blood flow reductions known to occur during headache episodes. ${ }^{6}$ Recent in vivo studies of the human brain using diffusion tensor (DT) MRI has unambiguously shown that in longstanding vascular conditions, such as leukoaraiosis ${ }^{7}$ and cerebral autosomal dominant arteriopathy with subcortical infarcts and leukoencephalopathy (CADASIL), ${ }^{8}$ there is a significant amount of tissue damage in the normal appearing brain tissue (NABT), which goes undetected when using conventional MRI.

To improve our understanding of white matter changes in patients with migraine and to investigate whether an association exists between the clinical phenomenology of the disease and an accurate estimate of the amount of brain damage, we measured, using DT-MRI and histogram analysis, ${ }^{9}$ the extent of damage of NABT in migraine patients with different clinical and conventional MRI phenotypes. In those patients with $\mathrm{T} 2$ visible abnormalities, we also assessed the correlation between the extent of macroscopic and "occult" tissue damage.

\section{PATIENTS AND METHODS}

We studied 34 patients (31 women and three men; mean age 35.2 years, range 18-55 years; mean duration of the disease 13.6 years, range $1-40$ years; mean number of episodes per year 44.6 , range $12-70$ ) with migraine..$^{10}$ All possible alternative diagnoses were carefully excluded. ${ }^{10}$ There were 28 patients affected by "migraine without aura" and six patients affected by "migraine with aura". ${ }^{10}$ Patients with hypertension, hypercholesteraemia, diabetes mellitus, vascular/ heart diseases, and other major systemic and neurological conditions were excluded. At the time of the MRI assessment, 11 patients were treated with prophylactic drugs. Seventeen sex and age matched healthy volunteers ( 15 women and two men; mean age 36.0 years, range $21-55$ years) served as controls. These controls had a normal neurological examination, had not suffered from migraine, and did not have any medical conditions that have the potential to result in white matter abnormalities on MRI scans. ${ }^{11}$ Local ethical committee approval and written informed consent from all the subjects were obtained before inclusion in the study.

Brain MRI scans were obtained using a 1.5 Tesla system. The following pulse sequences were acquired: (1) dual echo turbo spin echo $(\mathrm{TSE})(\mathrm{TR} / \mathrm{TE}=3300 / 16-98$, echo train length $=5)$; and (2) pulsed gradient spin echo (PGSE) echo planar (interecho spacing $=0.8 \mathrm{~ms}, \mathrm{TE}=123 \mathrm{~ms}$ ), with diffusion gradients applied in eight non-collinear directions, chosen in order to cover three dimensional space uniformly. ${ }^{12}$ For the TSE scans, 24 contiguous axial slices were acquired with $5 \mathrm{~mm}$ slice thickness, $256 \times 256$ matrix and $250 \times 250 \mathrm{~mm}$ field of view (FOV). The slices were positioned to run parallel to a line that joins the most inferoanterior and inferoposterior parts of the corpus callosum. ${ }^{12}$ For the PGSE scans, 10 axial slices with 5 mm slice thickness, $128 \times 128$ matrix and $250 \times 250 \mathrm{~mm}$ FOV were acquired, with the same orientation as the dual echo scans, with the second last caudal slice positioned to match exactly the central slices of the dual echo set.

Post-processing of MR data was performed by a single experienced observer, unaware to whom the scans belonged. Lesions were identified on the dual echo scans and lesion volumes were measured using a segmentation technique based on local thresholding. ${ }^{12}$ PGSE images were first corrected for geometrical distortion induced by eddy currents. ${ }^{12}$ Then, the diffusion tensor was calculated and mean diffusivity (MD) and fractional anisotropy (FA) derived for every pixel, as previously described. ${ }^{12}$ The diffusion images were interpolated to the same image matrix size as the dual echo, and then the $b=0$ step of the PGSE scans (T2 weighted, but not diffusion weighted) was co-registered with the dual echo T2 weighted images. ${ }^{12}$ Pixels containing extracerebral tissue (including the

Abbreviations: NABT, normal appearing brain tissue; $M D$, mean diffusivity; DT-MRI, diffusion tensor magnetic resonance imaging; FA, fractional anisotropy 
Table 1 Normal appearing brain tissue MD and FA histogram derived metrics from healthy volunteers and patients with migraine

\begin{tabular}{llll}
\hline & Healthy volunteers & Migraine patients & $\mathrm{p}^{*}$ \\
\hline Average MD (SD) $\left(\times 10^{-3} \mathrm{~mm}^{2} / \mathrm{s}\right)$ & $0.86(0.01)$ & $0.88(0.03)$ & $\mathrm{NS}$ \\
MD histogram peak height (SD) & $130.4(19.7)$ & $117.2(13.3)$ & 0.02 \\
Average FA & $0.22(0.01)$ & $0.22(0.01)$ & $\mathrm{NS}$ \\
FA histogram peak height & $44.7(4.8)$ & $46.4(5.8)$ & $\mathrm{NS}$ \\
\hline
\end{tabular}

*Student's $t$ test for unpaired data. MD, mean diffusivity; FA, fractional anisotropy

cerebrospinal fluid) were removed from the MD and FA maps, as previously described. ${ }^{12}$ Whenever needed, the final step consisted of automatic transfer of lesion outlines onto the MD maps and calculation of average lesion MD and FA. To study the MD and FA of NABT, pixels lying inside lesion outlines were nulled out, and $\mathrm{MD}$ and FA histograms of the remaining NABT were produced, as previously described. ${ }^{12}$ To correct for the between subject differences in brain volume, each histogram was normalised by dividing the height of each bin by the total number of pixels contributing to the histogram. For each histogram, the average MD and FA and the peak height were measured. Given the strong correlation existing between average histogram measures and the histogram peak location, ${ }^{13}$ the second quantity was not considered for this study, to reduce the risk of type I errors.

Student's $t$ test for unpaired data was used to compare MD and FA histogram derived metrics of the NABT between patients and controls and within patients with different clinical and MRI characteristics. Correlations were performed using the Spearman rank correlation coefficient.

\section{RESULTS}

White matter abnormalities were seen on the dual echo MRI scans from 14 patients with migraine $(40 \%)$. No lesions were detected on the dual echo scans from controls. In patients with migraine, median dual echo lesion load was $2.5 \mathrm{ml}$ (range $0.0-14.5 \mathrm{ml}$ ), average lesion MD $0.91 \times 10^{-3} \mathrm{~mm}^{2} / \mathrm{s}\left(\mathrm{SD} 0.03 \times 10^{-3}\right.$ $\mathrm{mm}^{2} / \mathrm{s}$ ), and average lesion FA 0.22 (SD 0.03 ).

In table 1 , the DT MRI histogram derived measures from the NABT from controls and patients with migraine are reported. Patients with migraine had lower MD histogram peak height than controls $(p=0.02)$ (fig 1$)$. No differences were found in FA histogram derived metrics between patients with migraine and controls. No difference in any MD and FA histogram derived metrics of the NABT was found between migraine patients with and without MRI lesions, and between migraine patients with and without aura (data not shown). In migraine patients with brain MRI abnormalities, average lesion MD was moderately correlated with MD histogram peak height of the NABT $(r=-0.45 ; \mathrm{p}=0.02)$.

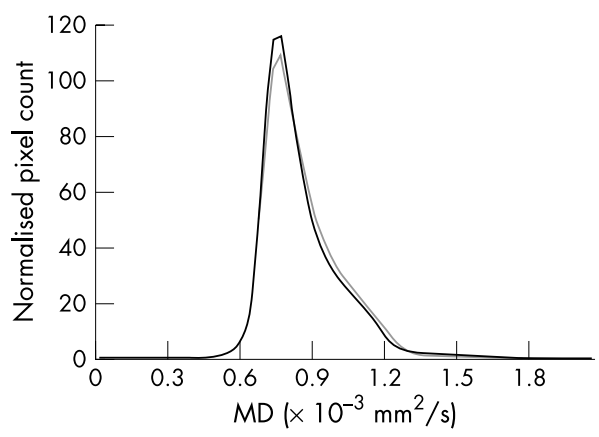

Figure 1 Mean diffusivity (MD) histograms of the normal appearing brain tissue from healthy subjects (black line) and patients with migraine (grey line).

\section{DISCUSSION}

Diffusion is the microscopic random translational motion of molecules, and water molecular diffusion can be measured in vivo using MR based technology. Because diffusion is affected by the properties of the medium where molecular motion occurs, measurements of diffusion inside biological tissues provide information about tissue structure at a microscopic level. ${ }^{14}$ DT MRI has been applied for the measurement of tissue damage in several neurological conditions, and has proved to have the potential to provide a more accurate picture of the pathological changes associated with vascular-9 15 and demyelinating ${ }^{12} 131617$ conditions than conventional T2 weighted imaging, as white matter areas classified as normal at the visual inspection of dual echo scans were found to be sites of subtle abnormality, ${ }^{7-9} 12131617$ which contributed to the clinical outcome. $^{781617}$

We obtained conventional dual echo and DT MRI scans of the brain from patients with migraine to assess the extent and the severity of tissue damage within and outside macroscopic white matter lesions. In agreement with previous studies, ${ }^{1-5}$ we found T2 visible lesions in $40 \%$ of the patients studied. We also measured MD of these lesions and found that, on average, MD was $3.4 \%$ higher than the MD of the NABT. In another study using the same DT-MRI sequence, ${ }^{16}$ we found that, on average, the MD values of MS lesions visible on dual echo scans is about $19 \%$ higher than that measured in normal appearing white matter with a SD that was more than three times higher than that found for lesions of the present study. The average difference in MD values between T2 visible lesions and normal appearing white matter was reported to be about $20 \%$ in patients with CADASIL $^{8}$ and about $30 \%$ in patients with leukoaraiosis. ${ }^{7}$ Similar considerations are also valid when comparing anisotropy of lesions with anisotropy of NABT in patients with migraine versus those with leukoaraiosis, ${ }^{7}$ CADASIL, ${ }^{8}$ and multiple sclerosis. ${ }^{16}$ All of this suggests that the amount of tissue damage in $\mathrm{T} 2$ visible lesions of migraineurs is comparatively mild compared with that of other neurological conditions, and probably does not have a great deal of impact on the daily life management of these patients.

The most intriguing and novel part of this study is, however, the finding that brain abnormality in migraine extends beyond T2 visible lesions and also involves the NABT. We found a reduction of the peak height of the NABT MD histograms in patients with migraine compared with controls. The peak height of the MD histogram is considered a measure of the residual amount of "truly" normal tissue, ${ }^{12}{ }^{18-20}$ as mild but widespread NABT damage is expected to increase the number of pixels with high MD values, thus causing a reduction of the peak height. The discrepancy of this finding with that of a previous MTR study, which was conducted on a different group of patients, is perhaps the result of a higher sensitivity of DT-MRI for the detection of ischaemic brain damage, ${ }^{78} 15$ than MT MRI. Although this study cannot provide clear cut answers about the nature of NABT abmormality in patients with migraine (but definitive histopathological studies are unlikely to be ever performed), there are at least two possible explanations for the reduced peak height of the NABT MD histogram found in these patients. Firstly, DT-MRI changes in 
the NABT might be the result of mild ischaemic insults with the potential to cause a loss of structural barriers to water molecular motion that can be detected by DT-MRI, but not by conventional MRI. Secondly, NABT changes in migraine might reflect secondary Wallerian degeneration of fibres traversing the macroscopically damaged tissue. Clearly, this second mechanism cannot be operative in patients with no T2 visible lesions, whereas the two mechanisms are not mutually exclusive in the remaining patients.

\section{Authors' affiliations}

M A Rocca, M Inglese, M Codella, M Filippi, Neuroimaging Research Unit, Department of Neuroscience, Scientific Institute Ospedale San Raffaele, University of Milan, Milan, Italy

B Colombo, G Comi, Department of Neurology, Scientific Institute Ospedale San Raffaele, University of Milan

Competing interests: none declared.

Correspondence to: Dr M Filippi, Neuroimaging Research Unit Department of Neuroscience, Scientific Institute Ospedale San Raffaele, Via Olgettina, 60, 20132 Milan, Italy; m.filippi@hsr.it

Received 28 July 2002

Accepted in revised form 23 November 2002

\section{REFERENCES}

1 Osborn RE, Alder DC, Mitchell CS. MR imaging of the brain in patients with migraine headaches. AJNR Am J Neuroradiol 1991;12:521-4.

2 Fazekas $F$, Koch $M$, Schmidt $R$, et al. The prevalence of cerebral damage varies with migraine type: a MRI study. Headache 1992;32:287-91.

3 Pavese N, Canapicchi R, Nuti A, et al. White matter MRI hyperintensities in a hundred and twenty-nine consecutive migraine patients. Cephalalgia 1994;14:342-5.

4 Cooney BS, Grossman RI, Farber RE, et al. Frequency of magnetic resonance imaging abnormalities in patients with migraine. Headache 1996;36:616-21.

5 Rocca MA, Colombo B, Pratesi A, et al. A magnetization transfer imaging study of the brain in patients with migraine. Neurology 2000;54:507-9.
6 Olesen J, Friberg L, Olsen TS, et al. Timing and topography of cerebral blood flow, aura, and headache during migraine attacks. Ann Neurol 1990;28:791-8

7 O'Sullivan $M$, Summers PE, Jones DK, et al. Normal-appearing white matter in ischemic leukoaraiosis: a diffusion tensor MRI study. Neurology 2001;57:2307-10.

8 Chabriat H, Pappata S, Poupon C, et al. Clinical severity in CADASIL related to ultrastructural damage in white matter: in vivo study with diffusion tensor MRI. Stroke 1999;30:2637-43.

9 van Buchem MA, Grossman RI, Armstrong C, et al. Correlation of volumetric magnetization transfer imaging with clinical data in MS Neurology 1998;50:1609-17.

10 Headache Classification Committee of the International Headache Society: Proposed classification and diagnostic criteria for headache disorders, cranial neuralgias and facial pain. Cephalalgia 1988;8 (suppl 7): 1-96.

11 Fazekas F, Barkhof F, Filippi M. Unenhanced and enhanced magnetic resonance imaging in the diagnosis of multiple sclerosis. J Neurol Neurosurg Psychiatry 1998;64:S2-5.

12 Cercignani $M$, Inglese $M$, Pagani $E$, et al. Mean diffusivity and fractional anisotropy histograms of patients with multiple sclerosis. AUNR Am J Neuroradiol 2001;22:952-8.

13 Rovaris M, Bozzali M, Santuccio G, et al. In vivo assessment of the brain and cervical cord pathology of patients with primary progressive multiple sclerosis. Brain 2001;124:2540-9.

14 Le Bihan D, Turner R, Moonen CT, et al. Imaging of diffusion and microcirculation with gradient sensitization: design, strategy, and significance. J Magn Reson Imaging 1991;1:7-28

15 de Crespigny AJ, Marks MP, Enzmann DR, et al. Navigated diffusion imaging of normal and ischemic human brain. Magn Reson Med 1995; 33:720-8.

16 Filippi $M$, Cercignani $M$, Inglese $M$, et al. Diffusion tensor magnetic resonance imaging in multiple sclerosis. Neurology 2001;56:304-11.

17 Filippi $M$, Tortorella $C$, Rovaris $M$, et al. Changes in the normal appearing brain tissue and cognitive impairment in multiple sclerosis. $J$ Neurol Neurosurg Psychiatry 2000;68:157-61.

18 van Buchem MA, McGowan JC, Kolson DL, et al. Quantitative volumetric magnetization transfer analysis in multiple sclerosis: estimation of macroscopic and microscopic disease burden. Magn Reson Med 1996;36:632-6.

19 Nusbaum AO, Tang CY, Wei T, et al. Whole-brain diffusion MR histograms differ between MS subtypes. Neurology 2000;54:1421-7.

$20 \mathrm{Ge} \mathrm{Y,} \mathrm{Grossman} \mathrm{RI,} \mathrm{Udupa} \mathrm{JK,} \mathrm{et} \mathrm{al.} \mathrm{Magnetization} \mathrm{transfer} \mathrm{ratio}$ histogram analysis of normal-appearing gray matter and normal-appearing white matter in multiple sclerosis. J Comput Assist Tomogr 2002;26:62-8 